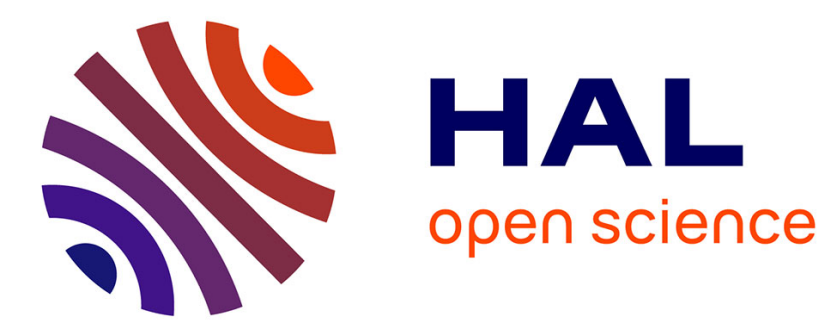

\title{
Turbo-like Iterative Thresholding for SAR Image Recovery from Compressed Measurements
}

\author{
Lei Yu, Yi Yang, Hong Sun
}

\section{To cite this version:}

Lei Yu, Yi Yang, Hong Sun. Turbo-like Iterative Thresholding for SAR Image Recovery from Compressed Measurements. 2009. hal-00384317

\author{
HAL Id: hal-00384317 \\ https://hal.science/hal-00384317 \\ Preprint submitted on 14 May 2009
}

HAL is a multi-disciplinary open access archive for the deposit and dissemination of scientific research documents, whether they are published or not. The documents may come from teaching and research institutions in France or abroad, or from public or private research centers.
L'archive ouverte pluridisciplinaire HAL, est destinée au dépôt et à la diffusion de documents scientifiques de niveau recherche, publiés ou non, émanant des établissements d'enseignement et de recherche français ou étrangers, des laboratoires publics ou privés. 


\title{
Turbo-like Iterative Thresholding for SAR Image Recovery from Compressed Measurements
}

\author{
Lei YU, Yi YANG and Hong SUN Member, IEEE,
}

\begin{abstract}
Compressive sensing (CS) has attracted many researchers since it offers a novel paradigm that one can acquire signals at a sub-Nyquist rate, and provides an implicit application of imaging and compressing simultaneously for SAR. This paper focuses on the recovery algorithms for compressed measurements of SAR data. Considering the characteristics of the SAR images, leading their not-very-sparsity in common representation space, a Turbo-like Iterative Thresholding algorithm based on Residual shrinkage operator (RTIT) is proposed. This algorithm aims at recovering signals which can not be very sparsely represented through some representation spaces. Some numerical results are presented to illustrate the performance of the proposed RTIT algorithm.
\end{abstract}

Index Terms-Compressive Sensing, Iterative Thresholding, Turbo.

\section{INTRODUCTION}

The recently developed technology - Compressive Sensing (CS), attracts many researchers. Unlike the traditional sampling methods, CS is a procedure with both sampling and compression, which permits signals being sampled at subNyquist rate and can be reconstructed from the compressed data [1] [2] [3]. The simple non-adaptive sampling principle and the high compressibility invoke a lot of potential applications in many different fields. In [4] [5] [6], authors have made a move to apply CS on remote sensing and obtained some great results in this field. Playing an important role on remote sensing, SAR has been widely used on earth observation, because of its properties of all weather and day-night imaging. However, the high-resolution and dynamic wide-range SAR images inevitably lead to enormous amount of data, which prescribes great burden on the limited-storage and low-speed sensors. In [6], a CS based SAR imaging system is addressed and the authors has given some preliminary results about the CS application on SAR.

However, sparsity, required as a prerequisite for CS theory [3], leads to a challenge of finding the sparse representation space for natural signals. Even more, the complicated structure of SAR images leads that the typical sparse representation space such as wavelet transform does not guarantee good sparse approximations in SAR images [6]. That is to say, the coefficients of SAR images over wavelet transform is not very sparse.

To date, many recovery algorithms based on convex optimization such as BP [7], and greedy algorithms such as OMP

This paper is supported by NSFC(60872131) and 863 National Project of China (2007AA12Z155).

The authors are with Signal Processing Laboratory, Electronic Information School, Wuhan University, China, 430079. Email: yuleiwhu@gmail.com, qingfeng4119@gmail.com, hongsun@whu.edu.cn.
[8], CoSaMP [9], are proposed, of which the hypothesis of known sparsity lever is requested. Whilst the natural signals may not satisfy this hypothesis even without noisy corruption. This results in the incomplete recovery if signals are not very sparse over a certain representation space.

In this paper, the improvements are considered simultaneously in two aspects. First, we apply shrinkage operator on the residual instead of the updated estimation to relax the constraint on the sparsity, and then use an iterative refinement of residual (RIT for abbreviation) to approximate the estimation. Theoretically and experimentally, this modification makes the recoveries for the not-very-sparse signals, including SAR images, much better.

Second, we turn to an over-complete dictionary to capture the complex structure of signals, i.e. to more sparsely represent signals. A turbo-like iterative scheme is adopted, where two RITs respectively with two irrelated representation spaces are introduced. The principle of RTIT is to use the compensation of one's to the other, to promote the information exchanges between the two RIT schemes, such as wavelet preserving smooth cartoons while curvelet preserving textures.

The following sections are organized as below. In section II, Compressive Sensing theory is briefly introduced. The classical Iterative Thresholding (IT) is introduced in section III. Section IV mainly discusses the scheme of Residual based Iterative Thresholding (RIT), based on which Turbolike Residual based Iterative Thresholding (RTIT) algorithm is proposed. In section $\mathrm{V}$, some numerical simulations are implemented to illustrate the proposed algorithms and experimentally prove their efficiency on SAR images. The paper is ended with a conclusion which gives some hints on the possible future works.

\section{Compressive Sensing}

According to compressive sensing theory (eg. [1]), a discrete signal or image expressed as a vector $f \in \Re^{n}$ can be exactly recovered with reduced measurements compared to the Nyquist rate provided that it is sparse in some basis, i.e. $f$ can be expressed as $f=\Psi x_{s}$, where $\Psi \in \Re^{n \times n}$ is a matrix whose columns are basis vectors and $x \in \Re^{n}$ is a $s$-sparse vector with only $s$ non-zero entries, $s \ll n$.

In CS framework, the measurements of images or signals are acquired from linear projections: $y=\Phi f$, where $\Phi \in$ $\Re^{m \times n}$ is measurement matrix with $m \ll n$ and $y \in \Re^{m}$ is the measurements. Given an arbitrary signal or image $f$ with $s$ Sparse representation $x_{s}$ over space $\Psi$, then the measurements $y$ of $f$ can be written as

$$
y=\Phi f=\Phi \Psi x_{s}
$$


In CS, the measurement matrix $\Phi$ can be randomly generated and then normalized by columns, such as $\Phi \sim \mathcal{N}(0,1)$. To recover signals from measurements $y$, one needs at least $m$ measurements with $m \geq \mathcal{O}(s \log n / s)^{1}$ [1]. Then the recovery of the signal or image $f$ is to find a sparsest signal $f^{*}$ in some basis $\Psi$ that matches the sensed measurements $y=\Phi f$, i.e.

$$
f^{*}=\arg _{g \in \Re^{n}} \min \|\Psi g\|_{l 0} \text { subject to } \Psi g=y
$$

The combinatorial optimization (2) is a NP-hard problem. And the convexification of the objective function is as the following formulation

$$
f^{*}=\arg _{g \in \Re^{n}} \min \|\Psi g\|_{l 1} \text { subject to } \Psi g=y
$$

Consider the noisy measurements $y=\Phi f+w$, where $w$ is a Gaussian noise of variance $\delta^{2}$, one can turn to the constrained form to solve (3)

$$
f^{*}=\arg _{g \in \Re^{n}} \min \frac{1}{2}\|\Phi g-y\|_{l 2}^{2}+\delta\|\Psi g\|_{l 1}
$$

where the first part is in some sense to be the error constraint and the second part is the sparsity constraint.

\section{ITERATIVE THRESHOLDING FOR SPARSITY MINIMIZATION}

Iterative Thresholding (IT) is a fast way to recover the signal from compressed measurements [11] [12], which aims at minimizing (4). This algorithm proceeds as follows, let $f^{(0)}=0$ and use the iteration

$$
f^{(i+1)}=H_{\sigma}\left(f^{(i)}+\lambda \Phi^{T}\left(y-\Phi f^{(i)}\right)\right)
$$

where $\lambda$ is the normalized parameter and $H_{\delta}(*)$ is the nonlinear shrinkage operator that keeps the larger coefficients, i.e.

$$
H_{\sigma}(a)=\Psi \text { Prune }_{\sigma}\left(\Psi^{-1}(f)\right)
$$

where $\Psi^{-1}(f)$ represents the inverse transformation, Prune $_{\sigma}(t)$ is a nonlinear operator that keeps the entries of $t$ larger than $\sigma$ and set the others to zero, i.e. Prune $_{\sigma}(t)=\operatorname{sign}(t)(|t|-\sigma)_{+}$. The algorithm is given in Algorithm 1.

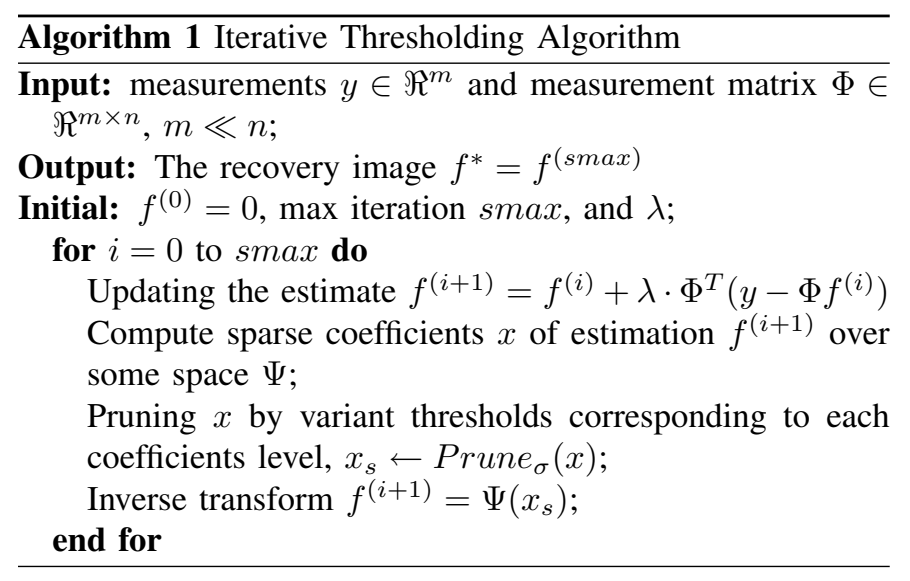

\footnotetext{
${ }^{1}$ This result is for Gaussian Random Measurements, but for Fourier Ensemble, it must be $m \geq \mathcal{O}\left(s(\log n / s)^{6}\right)$, see [10] for details.
}

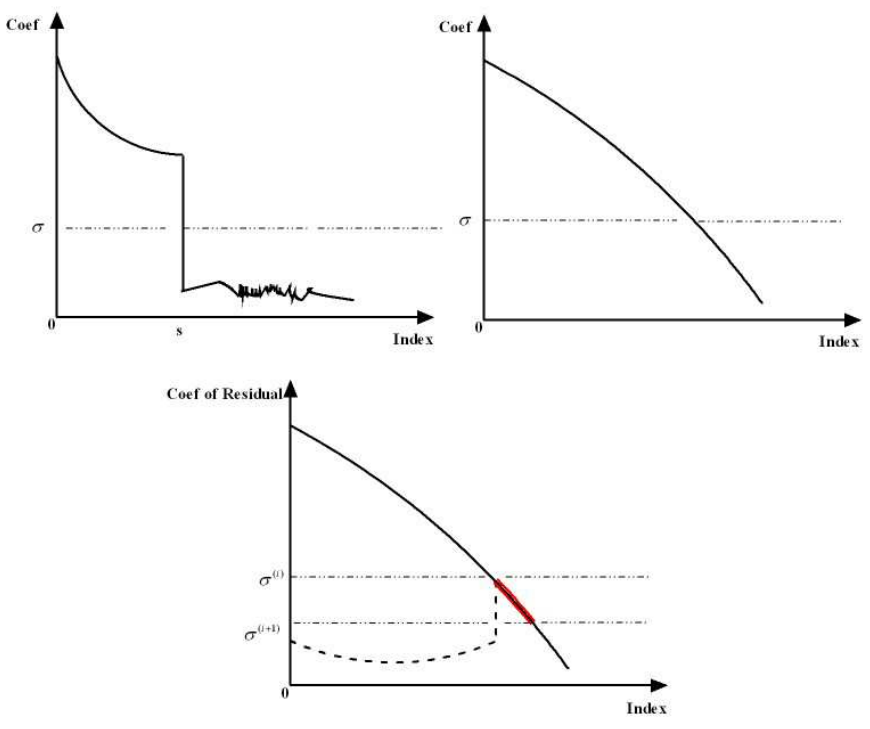

Fig. 1. [Top-left] The representation coefficients of a $s-$ Spar se signal; [topright] The representation coefficients of a not-very-sparse signal; [bottom] the representation coefficients of the residual at iteration $i$ of RIT.

Remark 1: In Algorithm 1, the threshold $\sigma$ of the pruning function $\operatorname{Prune}_{\sigma}(*)$ can be set as a descending variable to obtain better performance. And if the original signal is strictly sparse over a fixed representation space, the recovery of IT will be very good, as shown in Fig. 1.

\section{Proposed Algorithm}

\section{A. Motivations}

The existed algorithms for CS recovery such as BP, OMP are based on the $s-$ Sparse hypothesis of signals. However, the noise-like properties and wide dynamical range data of SAR images leads that typical representation space such as wavelet transform does not guarantee good sparse approximation for SAR images. That is to say SAR images are not very sparse over the existed typical representation spaces, as shown in top-right of Fig. 1. Thus, the first problem of recovery for SAR from compressed measurements is how to reconstruct through not-very-sparse representation.

On the other hand, fixed representation space are not flexible enough to capture the complex redundancy of natural images. For instance the wavelet transform lacks of translation and rotation invariance and is not efficient to compress geometric images [12]. Here is the second problem that how to capture information as completely as it can.

In the rest of this section, respectively, two modifications for IT are taken into account targeting on solving the above two problems.

\section{B. Residual Based Iterative Thresholding algorithm}

First, we give the notation of Residual $r^{(i)}=\Phi^{T}\left(y-\Phi f^{(i)}\right)$ which represents the remained information of each iteration. Then some variations on (5) are presented, written as

$$
f^{(i+1)}=f^{(i)}+\lambda H_{\sigma}\left(r^{(i)}\right)
$$


where the nonlinear shrinkage operator is just over the residual $r^{(i)}$, instead of the updated estimation $f^{(i)}$ and $\lambda$ is the weight of each refinement that can be constant value or a variant parameter. The detailed algorithm is given in Algorithm 2. Obviously, the main difference between IT and RIT is the shrinkage object - shrink on estimation for IT and shrink on residual (Importance Refinement) for RIT. Here is the comparison of the two shrinkage operators.

\section{Commonness}

Both shrinkage operators aim at keeping the important information while eliminating the less important information (noises), which is reflected on the value of the coefficients.

\section{Differences}

For IT, the shrinkage operator works on updated estimation at each iteration, shown in top figures of Fig. 1. The coefficients larger than threshold $\sigma$ are preserved while ones smaller than $\sigma$ are eliminated. This kind of procedure will result in fast convergence and good results for signals, when signals are strictly $s-$ Sparse, as shown in top-left of Fig. 1. But for some signals with coefficients like the top-right of Fig. 1, some minor information will be omitted.

However, for RIT, the shrinkage operator is on residual at each iteration, shown in bottom figure of Fig. 1. Since the larger coefficients at the previous iteration are subtracted, the relatively minor large coefficients (shown as the red line in bottom figure of Fig. 1), which may contain some important information, will be preserved at the current iteration.

From above analysis, we can make a conclusion that RIT works much better than IT for recovery of not-very-sparse signals, because in some instances, the most significant coefficients of the image may not correspond to the largest values. In RIT, it may then happen that the image possesses significant coefficient inside the cut-off, whilst it will not happen in IT.

Remark 2: The convergence of RIT will be slower than IT, since the refinement cannot be very coarse in case of the overrefinement (see Fig 4).

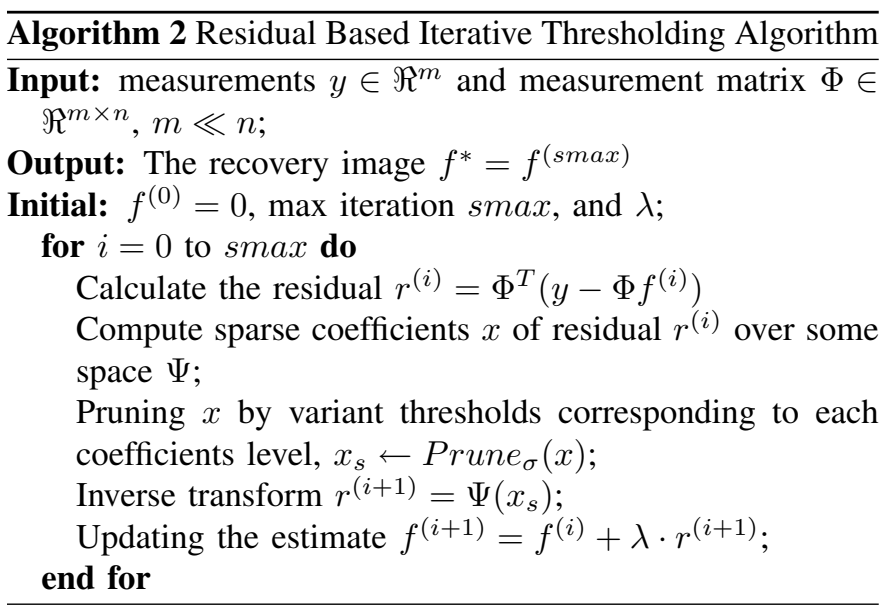

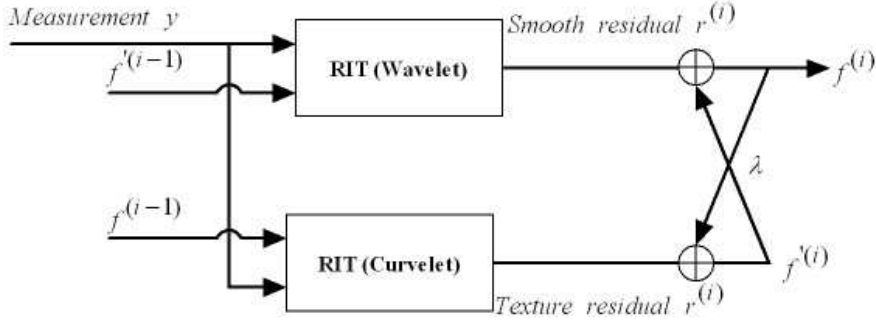

Fig. 2. The Scheme of Residual based Turbo-like Iterative Thresholding Algorithm

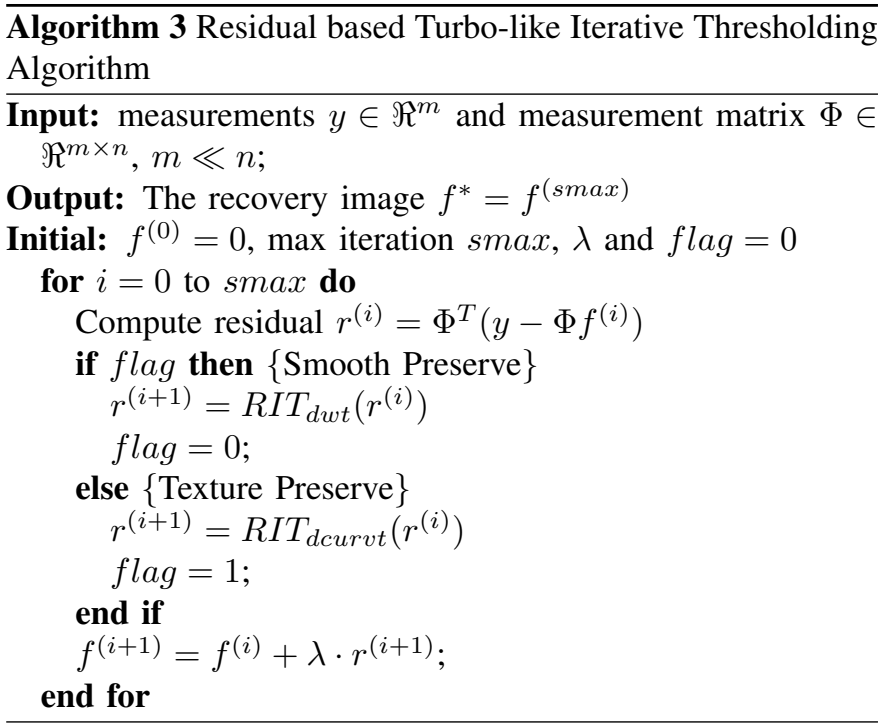

\section{Residual based Turbo-like Iterative Thresholding (RTIT)}

To solve the second problem, we turn to choosing an over-complete dictionary [13], which contains at least two irrelated representation spaces. And then, turbo-like iterative scheme is adopted to combine the multi-RIT iterative scheme. In [14] [15] [16], the authors have proposed a turbo iterative signal processing paradigm, which has been applied in speech enhancement[14], image filter[15], and radar image processing[15]. From the global view, turbo iterative enforces the propagation of the information exchange between the two irrelated models [14], which promotes the performance of recovery. ${ }^{2}$

As shown in Fig. 2, obviously, the information exchanges are carried out through tubo-like procedure. And for instance, wavelet shrinkage and curvelet shrinkage are chosen as the two irrelated model, of which the former preserves the smooth cartoons but omits the textures, while the later works oppositely. The iterative process can be considered as compensations between the two irrelative models. The detailed algorithm of RTIT is addressed in Algorithm. 3.

\section{NumericAl RESUlts}

In order to evaluate the performance of the proposed algorithm, the numerical simulations are carried out on optical images. And then, we implement RTIT on raw SAR data,

\footnotetext{
${ }^{2}$ It will not be proven here, but intuitively and experimentally, it is true.
} 
which contains some special features, for instance bright point, regular grids, and complex structural urban areas.

The compressed measurements in the following experiments are generated through Furier ensemble ${ }^{3}$, and then corrupted by a Gaussian noise $w \sim \mathcal{N}(0, \delta)$, where $\delta=5(5 \%)$ for optical images $^{4}$. In Algorithm 1 and 2, the sparse representation space is chosen as wavelet. For the initialization of 3 algorithms, the thresholds are variant with the sparse coefficients, where $\sigma=\operatorname{median}(x) / 0.6745 * \nu$ for wavelet coefficients in Algorithm 1 and $\sigma=\operatorname{median}(x) / 0.6745 * 2.5$ for coefficients in Algorithm 2 and 3 , where $\nu$ is a descending value variant from 0.5 to 0.1 . $\lambda$ is fixed as 1 in all experiments.

\section{A. Experiments on Optical Images}

The objective of this experiment is to illustrate the outstanding performance of RIT and RTIT. As shown in Fig. 4, with the same sample rate $(48.83 \%)$, all 3 algorithms can approximately recover the original image very well. However, the residual error, obtained through the difference between the original image and its recovery, shows that almost all texture information is preserved at the RTIT recovery, the RIT performs little worse but the IT just can preserve the smooth cartoons and some very simple textures. Furthermore, the PSNR of the recovery also shows the outstanding performance of RIT and RTIT, of which RTIT converges much faster than RIT due to the Turbo-like information exchanges.

Fig. 5 shows the performances of the 3 algorithms with different sampling rates, which are $12.21 \%, 24.41 \%, 48.83 \%$ and $73.24 \%$. Without loss of generality, the test image is also extracted from the Barbara image. All experiments are proceeded the same 200 iterations. Obviously, the results shows that with the same sampling rate, RTIT can converge to the most optimal solution among the 3 algorithms, RIT performs little worse, and IT is worst.

\section{B. Experiments on SAR Images}

In this simulation, the test SAR images are extracted from the TerreSAR-X data and all the test images are with size of $512 \times 512$. Referred to [6], the Fourier Ensemble random sampling is adapted to obtain the synthesis measurements, with the sampling rate $38.15 \%(10,0000$ samples). Since the restriction of the paper, the comparison between the algorithms will not be presented, instead of the recovery results of RTIT, as shown in Fig. 6. Respectively, SAR images with bright point, regular grid, and complex urban areas are considered in this simulation. Visually, RTIT is with very good performance, where almost all information of the original SAR images are preserved. To evaluate the performance of the recovery qualitatively, the pseudo-PSNR is introduced as

$$
P S N R_{\text {pseudo }}=10 \log \frac{\|f\|_{2}^{2}}{\left\|f-f^{*}\right\|_{2}^{2}}
$$

where $f$ is the original SAR image and $f^{*}$ is its corresponding recovery. Tab. I presents the pseudo-PSNR of four different

\footnotetext{
${ }^{3}$ That's why DCT can't be used here for sparse representation matrix.

${ }^{4}$ There is no noise corruption on the measurement of SAR images, since the data itself is with noisy property.
}

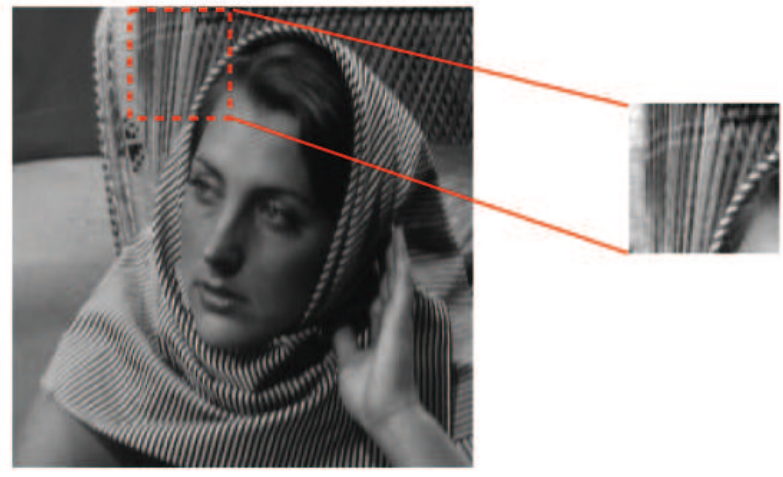

Fig. 3. The original Barbara image.
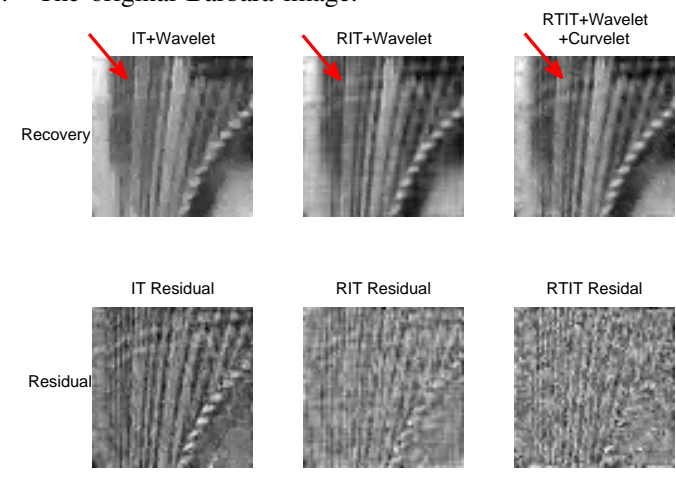

RTIT Residal
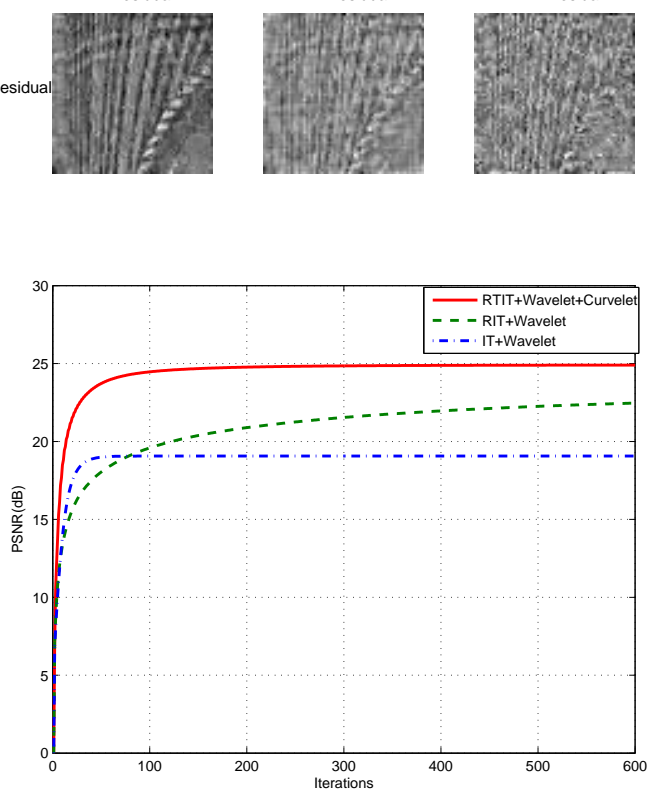

Fig. 4. [Top]The recovery of the partial image shown in Fig 3 through IT, RIT and RTIT respectively; [middle] the residual error between the original image and its recovery; [bottom] the iterations versus PSNR.(with sample rate $48.83 \%$.)

types of SAR images along with the iterations, where the complex urban areas are with the best recovery performance.

TABLE I

THE PSEUDO-PSNR OF RECOVERIES FOR SAR IMAGES(DB).

\begin{tabular}{l|ccccc}
\hline \hline Iterative numbers & 10 & 20 & 50 & 100 & 200 \\
\hline Bright point & 7.629 & 10.53 & 14.23 & 16.9 & 19.42 \\
\hline Block areas & 5.876 & 7.904 & 10.92 & 13.49 & 17.58 \\
\hline Regular grids & 9.158 & 12.4 & 16.38 & 19.05 & 21.21 \\
\hline Complex urban & 11.08 & 14.49 & 18.58 & 21.52 & 23.44 \\
\hline
\end{tabular}



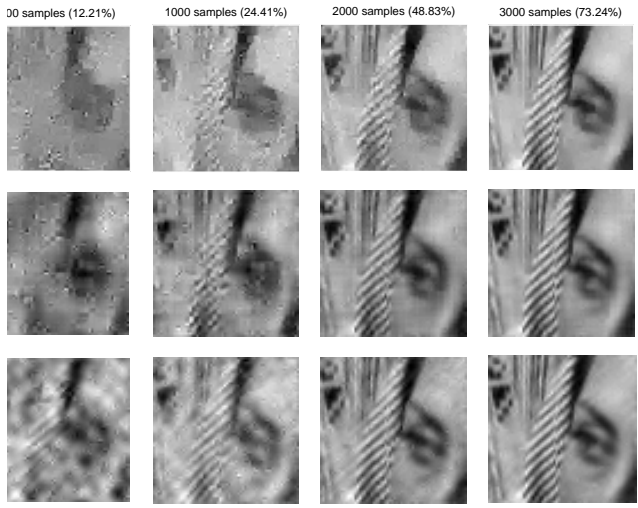

Fig. 5. The recoveries for the partial of the image Fig 3. From left to right IT, RIT and RTIT algorithms are implemented with the sample rate $12.21 \%$, $24.41 \%, 48.83 \%$ and $73.24 \%$
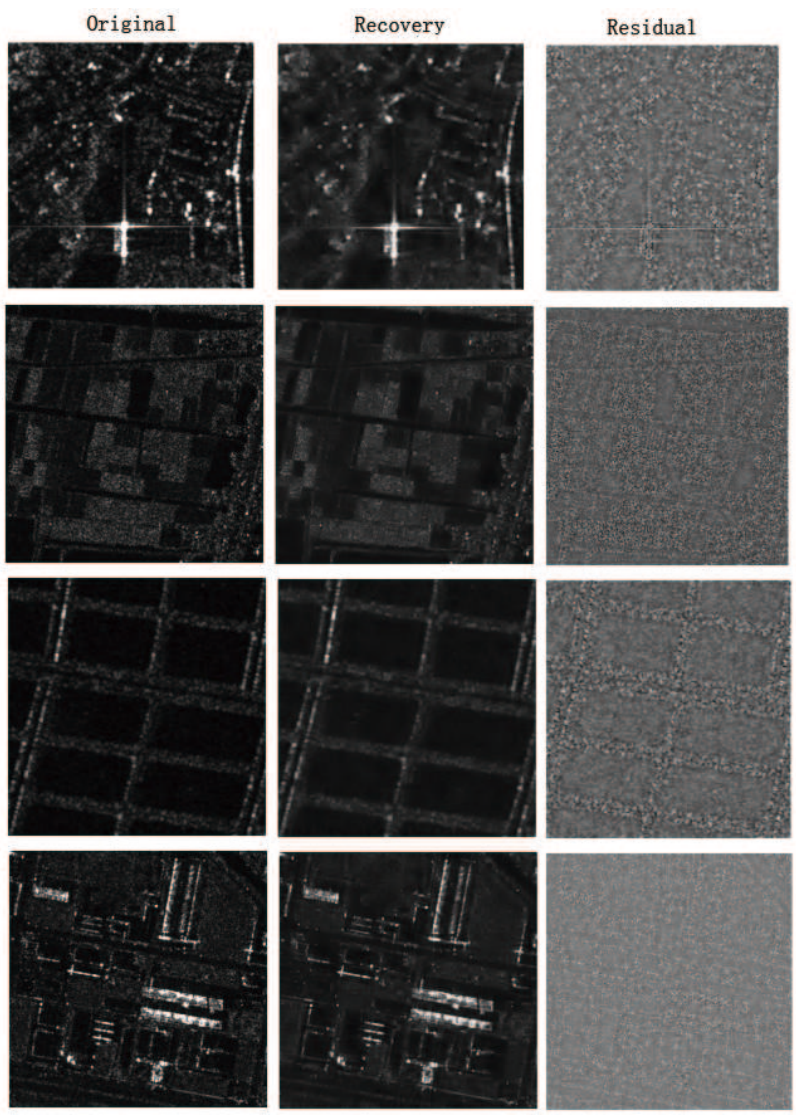

Fig. 6. RTIT algorithm implemented on SAR images. From top to bottom, each level of images respectively represent a class of scenes, i.e., bright point, block areas, regular grids and complex urban areas. From left to right, the columns are respectively original SAR images, recoveries, and residual errors.

\section{CONCLUSION}

The main contribution of this paper is that a Turbo-like Iterative Thresholding based on Residual shrink (RTIT) is proposed. And we experimentally prove the better performance of RTIT when recovering the not-very-sparse images over wavelet transform. Meanwhile, the preliminary results on SAR images show the potential application of Compressive Sensing (CS) on SAR compression.

In the numerical simulation, only two sparse representation spaces are used - wavelet and curvelet. It might be much better to use more sparse representation spaces such as Bandelets [17], Countourlets [18], Directionlet [19], inside the RTIT to preserve more features. The other open problem of this paper is the proof of the convergency of RIT and RTIT, which will be the future work.

\section{REFERENCES}

[1] R. Baraniuk, "Compressive sensing," in Proc. 42nd Annual Conference on Information Sciences and Systems CISS 2008, 19-21 March 2008, pp. iv-v.

[2] R. G. Baraniuk, "Compressive sensing [lecture notes]," IEEE Signal Processing Mag., vol. 24, no. 4, pp. 118-121, July 2007.

[3] E. J. Candes and M. B. Wakin, "An introduction to compressive sampling," IEEE Signal Processing Mag., vol. 25, no. 2, pp. 21-30, March 2008.

[4] J. Ma, "Single-pixel remote sensing," IEEE GEOSCIENCE AND REMOTE SENSING LETTERS, vol. 6, no. 2, pp. 199-203, April 2009.

[5] R. Baraniuk and P. Steeghs, "Compressive radar imaging," in Proc. IEEE Radar Conference, 17-20 April 2007, pp. 128-133.

[6] M. D. Gabriel Rilling and B. Mulgrew, "Compressed sensing based compression of sar raw data," in SPARS'09 - Signal Processing with Adaptive Sparse Structured Representations (2009), 2009.

[7] E. J. Candes and T. Tao, "Decoding by linear programming," IEEE Trans. Inform. Theory, vol. 51, no. 12, pp. 4203-4215, Dec. 2005.

[8] J. A. Tropp and A. C. Gilbert, "Signal recovery from random measurements via orthogonal matching pursuit," IEEE Trans. Inform. Theory, vol. 53, no. 12, pp. 4655-4666, Dec. 2007.

[9] D. Needell and J. A. Tropp, "Cosamp: Iterative signal recovery from incomplete and inaccurate samples," Appl. Comput. Harmon. Anal., to appear.

[10] E. J. Candes and T. Tao, "Near-optimal signal recovery from random projections: Universal encoding strategies?" IEEE Trans. Inform. Theory, vol. 52, no. 12, pp. 5406-5425, Dec. 2006.

[11] M. D. I. Daubechies and C. D. Mol, "An iterative thresholding algorithm for linear inverse problems with a sparsity constraint," Commun. Pure Appl. Math., vol. 57, no. 11, pp. 1413-1457, Nov. 2004.

[12] G. Peyré, "Best basis compressed sensing," Proc. SSVM, pp. 80-91, June 2007.

[13] B. A. Olshausen and D. J. Field, "Sparse coding with an overcomplete basis set: A strategy employed by v1," Vision Research, vol. 37, 1997.

[14] H. Sun and H. Maitre, "Turbo iterative signal processing," in Proc. IEEE 13th Digital Signal Processing Workshop and 5th IEEE Signal Processing Education Workshop DSP/SPE 2009, 4-7 Jan. 2009, pp. 495-500.

[15] H. Sun, H. Maitre, and B. Guan, "Turbo image restoration," in Proc. Seventh International Symposium on Signal Processing and Its Applications, vol. 1, 1-4 July 2003, pp. 417-420.

[16] G. Farage, S. Foucher, C. Lopez-Martinez, and G. B. Benie, "Turbo speckle filtering applied to polsar data," in Proc. IEEE International Geoscience and Remote Sensing Symposium IGARSS 2008, vol. 4, 7-11 July 2008, pp. IV-25-IV-28.

[17] M. S. Le Pennec E., "Sparse geometric image representations with bandelets," IEEE Transactions on Image Processing, vol. 14, no. 4, pp. 423-438, 2005

[18] M. N. Do, "Contourlets and sparse image representations," SPIE Conference on Wavelet Applications in Signal and Image Processing X, San Diego, USA, August 2003.

[19] V. Velisavljevic, B. Beferull-Lozano, M. Vetterli, and P. L. Dragotti, "Directionlets: anisotropic multidirectional representation with separable filtering," IEEE Trans. Image Processing, vol. 15, no. 7, pp. 1916-1933, July 2006. 